\title{
FDTD Simulation of the Nonlinear Gain Dynamics in Active Optical Waveguides and Semiconductor Microcavities
}

\author{
Gabriela M. Slavcheva, John M. Arnold, and Richard W. Ziolkowski, Fellow, IEEE
}

\begin{abstract}
In this paper, we use the finite-difference time-domain (FDTD) solution of the full-wave vectorial Maxwell-Bloch equations for a two-level quantum system developed earlier [18], [19] to investigate the nonlinear gain spatio-temporal dynamics of active optical waveguides and semiconductor microcavities. The numerical model has been successfully validated against density matrix theory of gain saturation in homogeneously broadened two-level quantum systems for optical waveguides containing resonant gain nonlinearities. The semiclassical equations have been extended employing the Langevin formalism to account for the quantum noise and the spontaneous emission. We have numerically demonstrated the time evolution of the coherent oscillations build up at the output laser facet identifying the lasing threshold and the fast relaxation oscillations until the settlement of a steady-state emission. Our simulation predictions of the lasing wavelength in a number of vertical-cavity surface-emitting laser geometries, when the spontaneous emission is the only source of radiation, agree very well with standard results and, thus, allow us to infer and subsequently optimize important emission characteristics, such as the spontaneous emission rate, the laser line shape, and the relaxation oscillation frequencies and decay rates.
\end{abstract}

Index Terms-Finite-difference time-domain (FDTD) method, Maxwell-Bloch system, nonlinear gain dynamics, quantum noise, semiconductor microcavities, spontaneous emission.

\section{INTRODUCTION}

$\mathbf{R}$ ECENT advances in several integrated semiconductor optoelectronics technologies along with the development of high-speed optical communication systems have been increasing the demand for optimized ultrashort light pulse designs, sources, and application-driven devices. In this respect, the modeling of the spatiotemporal dynamics, or specifically the dynamic response of novel ultrafast integrated devices on ultrashort time scales, becomes increasingly important for successfully optimizing the performance of these devices. It is anticipated that optimized design methodologies will be able to direct semiconductor microcavity laser mode-locking techniques toward an objective of integrated ultrashort pulse generation in vertical-cavity surface-emitting lasers (VCSELs).

Manuscript received December 15, 2003; revised July 26, 2004.

G. M. Slavcheva is with the Advanced Technology Institute, School of Electronics and Physical Sciences, Physics Department, University of Surrey, GU2 7XH Surrey, U.K. (e-mail: G.Slavcheva@surrey.ac.uk).

J. M. Arnold is with the Department of Electronics and Electrical Engineering, Optoelectronics Research Group, University of Glasgow, G12 8QQ Glasgow, U.K. (e-mail: jma@elec.gla.ac.uk).

R. W. Ziolkowski is with the Department of Electrical and Computer Engineering, University of Arizona, Tucson, AZ 85718 USA (e-mail: ziolkowski@ ece.arizona.edu).

Digital Object Identifier 10.1109/JSTQE.2004.836023
The spatiotemporal dynamics of semiconductor laser devices has been successfully simulated by the rate equations approach [1]. However, even though these results have been proven to be extremely useful, the rate equations have well-known limitations for their applicability. They are generally not valid for pulse durations much shorter than the characteristic lifetimes in the medium. Furthermore, the gain medium cannot be assumed to respond instantaneously on a femtosecond time scale to the optical excitation; and, consequently, the induced polarization cannot be eliminated adiabatically.

Most of these limitations can be overcome with the more general formalism of the semiclassical Maxwell-Bloch equations that employs the density-matrix method (see, e.g., [2]-[4], and the references therein). Because of the complexity of this treatment, the associated optical field equations have been traditionally simplified using several approaches. These include the rotating wave approximation (RWA), the slowly-varying envelope approximation (SVEA), and splitting the optical field into counter-propagating waves [5]-[7]. The approximations associated with these methods limit their range of applicability when ultrashort pulse phenomena and inhomogeneous media are considered and, consequently, these methods are unable to account for the complex dynamics of ultrafast systems.

Our aim in this paper, is to extend the modeling of the nonlinear optical waveguide and microcavity structures beyond the limits of the commonly applied approximations, exploiting the full-wave vectorial form of Maxwell-Bloch equations. Undertaking such an effort is justified by the recent experimental and theoretical studies of the ultrashort pulse propagation in semiconductor optical amplifiers (SOAs) [8], [34], [35] and the transient response investigations under femtosecond pulse excitations in VCSELs [9], [36]. These investigations definitely show that new physical effects become important in this regime which require treatments beyond the usual approximations [10], [11]. We have demonstrated the new features present in the new full-wave Maxwell-Bloch solution in tackling the self-induced transparancy (SIT) problem in one- (1-D) [18] and two-dimensions (2-D), [32], [33] that are absent in the standard approximations. The time derivatives of the electric field of the SIT pulses play an essential role in the nonlinear evolution of the quantum system. These effects have been confirmed by other authors (see [11] and references therein).

Modeling the spatiotemporal dynamics of semiconductor lasers beyond the limits of applicability of the previously mentioned formalisms represents a challenging task. Moreover, progress in integrated optoelectronic technologies has 
reduced laser device dimensions to be on the order of a single wavelength in size. As a consequence, the quantum fluctuations in the light field become increasingly important. Therefore, a comprehensive model of the quantum noise effects is indispensable for the correct simulation of the optical field evolution.

There have been a number of approaches in the literature for a fully consistent inclusion of the quantum fluctuations of the light field in the dynamics of the semiconductor microcavity lasers. The nonequilibrium Green's function formalism suggested in the early nineties [12], [13] has provided a rigorous framework for treatment of the quantum noise. However, a major drawback of this approach is the greater computational effort associated with the Green's function calculation as compared to that for integration of the corresponding Maxwell-Bloch equations. An alternative approach to the rigorous treatment of the quantum fluctuations and the spontaneous emission and amplified spontaneous emission in semiconductor laser devices is based on Maxwell-Bloch equations in second quantization form, exploiting the quantum operator dynamics of the field, carrier system and the local light-matter dipole interaction [14]. It has been shown that the spontaneous emission term originates from the dipole-dipole correlations. A fully quantum-mechanical analysis of the interacting electron-hole photon system has been developed and implemented in a series of papers by Kira et al. [15]-[17]. This method is based on a quantum form of the semiconductor Bloch equations (SBEs) through the Heisenberg equations for the operator dynamics of the microscopic polarization, carrier occupation, and the photon operator of the quantized light field. This results in a set of semiconductor luminescence equations which reduce to the SBE in the semiclassical limit. Thus, the spontaneous emission term can be identified as a source of the recombination process and acts as a source term for the field-dipole correlations.

We should note, however, that it is possible to derive quantum mechanical Langevin equations for the two-time correlation functions of quantum statistics directly from the quantum mechanical operator dynamics [20], [21]. It has been demonstrated [20] that the operator equations of motion can be replaced by a c-number Fokker-Planck equation, which, in turn, can be written in an equivalent set of "classical" c-number Langevin equations. This yields an associated "classical" random process that contains all the quantum information. The diffusion coefficient in the Fokker-Planck equation demonstrate the shot-noise origin of the fluctuations. This has lead to the discovery of the principle of the correspondence between the quantum noise effects and the classical noise effects.

In this paper, we exploit the quantum-classical correspondence in the presence of quantum noise by formulating stachastic equations simulating the quantum noise by adding random (Langevin) noise terms to the deterministic evolution of the optical field and medium polarization. Therefore, we shall neglect the quantization effects in our formulation and will represent the fields, polarization and carrier populations as classical variables rather than as operators. We introduce a generalization of the complete semiclassical treatment that generates the optical field by solving Maxwell's equations in vector form simultaneously with the time-evolution equations for a two-level quantum system given in [18] and [19]. Analogous to the stochastic rate equations formalism (see, e.g., [22], [23]), we have introduced a random electric field fluctuation noise term into the Maxwell-Bloch system through the Maxwell's equations. This term generates the statistical fluctuations of the laser field which, in turn, induce fluctuations in the population inversion, thus modifying the equations of motion of the quantum system. Using this extended Maxwell-Bloch equation set, we investigate the nonlinear spatiotemporal dynamics of active optical waveguides and semiconductor microcavities containing resonant gain nonlinearities.

In Section II, we discuss the theoretical background of our extension and introduce its numerical implementation. In Section III, we apply our extended Maxwell-Bloch approach to several configurations including a slab optical waveguide that contains a resonant gain nonlinearity and an active semiconductor microcavity. Reproduction of known approximate results in the appropriate limits for these configurations is emphasized to validate our approach. We summarize our results in Section IV.

\section{THEORETICAL BACKGROUND AND NumericAL IMPLEMENTATION}

Our theoretical considerations are based on the coherent optical Maxwell-Bloch equations for a two-level quantum system in real-vector representation of the density matrix [18], [19]. The Maxwell-Bloch equations defined for a two-level system having a resonant transition frequency $\omega=\omega_{0}$, including the damping of the resonant dipoles, and for a plane wave propagating along the $z$ axis that is polarized along the $x$ axis, have the form

$$
\begin{aligned}
\frac{\partial H_{y}}{\partial t} & =-\frac{1}{\mu} \frac{\partial E_{x}}{\partial z} \\
\frac{\partial E_{x}}{\partial t} & =-\frac{1}{\varepsilon} \frac{\partial H_{y}}{\partial z}-\frac{N_{a} \wp}{\varepsilon T_{2}} \rho_{1}+\frac{N_{a \wp} \omega_{0}}{\varepsilon} \rho_{2} \\
\frac{\partial \rho_{1}}{\partial t} & =-\frac{1}{T_{2}} \rho_{1}+\omega_{0} \rho_{2} \\
\frac{\partial \rho_{2}}{\partial t} & =-\omega_{0} \rho_{1}-\frac{1}{T_{2}} \rho_{2}+2 \frac{\wp}{\hbar} E_{x} \rho_{3} \\
\frac{\partial \rho_{3}}{\partial t} & =-2 \frac{\wp}{\hbar} E_{x} \rho_{2}-\frac{1}{T_{1}}\left(\rho_{3}-\rho_{30}\right)
\end{aligned}
$$

where $\rho$ is a real three-vector (the Bloch vector) in the Hilbert space describing the time evolution of the quantum system. The real vector components are related to the elements of the density matrix by the following relations:

$$
\begin{aligned}
& \rho_{1}=\hat{\rho}_{12}+\hat{\rho}_{21} \\
& \rho_{2}=i\left(\hat{\rho}_{12}-\hat{\rho}_{21}\right) \\
& \rho_{3}=\hat{\rho}_{22}-\hat{\rho}_{11} .
\end{aligned}
$$

The physical meaning of the real Bloch vector components is straightforward. The components $\rho_{1}$ and $\rho_{2}$ represent, respectively, the dispersive or in-phase and the absorptive or in-quadrature polarization components associated with the dipole transition $1 \rightarrow 2$. The term $\rho_{3}$ represents the fractional population difference in the two-level system states 1 and 2. In the Maxwell-Bloch equation set, the dissipation in the system has 
been accounted for by the introduction of the phenomenological relaxation times $T_{1}$ and $T_{2}$. They represent, respectively, the population relaxation (longitudinal) time and the dephasing (transverse) time. We have used the following notations: $N_{a}$ is the resonant dipole density (number of polarizable atoms (oscillators or excitons) per unit volume) and $\wp=e q_{0}$ is the dipole coupling coefficient, where $q_{0}$ is a measure of the separation between the charges in the dipole. The equilibrium fractional population difference $\rho_{30}$ in (5) is the initial population profile of the two-level system. We will assume that $\rho_{30}=-1$ corresponds to a system which is initially in its ground state and $\rho_{30}=1$ to the system with population inversion created by some pumping process, i.e., initially in the excited upper state. Thus, we can model both resonant absorption and gain media within the two-level approach.

The full-wave vector Maxwell's equations coupled to the time evolution equations of the two-level quantum system are discretized using the Yee, staggered-grid finite difference algorithm for Maxwell's equations [24]. The system is solved numerically in the time domain using a predictor-corrector iterative procedure at each time step by the standard leapfrog time-advancing scheme (FDTD) [18], [19]. At each time step, the stability criterion (Courant condition): $c \Delta t / \Delta z \leq 1$, is fulfilled. We have used very fine discretization in space $\Delta z=1 \AA$ in order to spatially resolve the quantum wells of the VCSEL structures, which represents $\lambda / 8500$ to $\lambda / 13000$ for the structures considered. The corresponding time step was a small $\left(\sim 10^{-4}\right)$ fraction of a femtosecond. With this discretization, we have found that the iterative scheme implemented converges and within 25 iterations gives differences between the new and the old values that are smaller than $1 \times 10^{-5}$. The chosen predictor-corrector scheme has been thoroughly investigated in terms of its ability to satisfy the lossless-case constraint $\rho_{1}^{2}+\rho_{2}^{2}+\rho_{3}^{2}=1[18]$ and proved to be very efficient even applied to the much more computationally demanding 2-D problem [32], [33].

This numerical approach allows a very general treatment that properly accounts for the nonlinearity, dispersion, absorption/amplification, saturation and resonant effects without invoking any of the standard approximations (such as the SVEA or RWA). It thus allows a complete description of the interaction of an ultrashort optical pulse with an ultrafast medium.

The time evolution of a two-level quantum system in an external electric field represents a Goursat-type initial boundary value problem that is well posed if the whole time history of the initial electric field is given along some characteristic (e.g., the beginning of the simulation domain $z=0$; see, for instance, [25]). The boundary conditions imposed at the left and right boundaries of the 1-D FDTD domain are based on the exact one-way wave equation approach, yielding a second-order accurate difference scheme throughout the simulation region. We have estimated the reflection error associated with the transmitting boundary conditions and the discretization choice to be between 100 and $120 \mathrm{~dB}$ smaller than the peak signal strength.

Generally, a source pulse with predesigned shape and carrier frequency resonant with the transition frequency of the quantum system is excited at the left boundary (or at any point within the simulation domain) and its propagation through the medium is sampled at given points across the structure as a function of time. We have identified a continuous-wave sinusoidal pulse with carrier frequency $\omega_{0}$, smoothly switched on from 0 to maximum amplitude within five periods, as a particularly suitable pulse shape for the purpose of investigation of the nonlinear gain effects in optical slab waveguides

$$
E_{x}(t)=E_{0} \sin \left(\omega_{0} t\right)\left\{\begin{array}{ccc}
0, & \text { for } & t<0 \\
\left(1-x^{2}\right)^{4}, & \text { for } & 0 \leq t \leq 5 T_{p} \\
1, & \text { for } & t>5 T_{p}
\end{array}\right\}
$$

with $x=\left(t-5 T_{p}\right) / 5 T_{p}, E_{0}$ being the maximum amplitude of the initial pulse, and $T_{p}$ being the period of one cycle of the sinusoid. We have used random white Gaussian noise generated within the cavity for our studies of the coherent response of the semiconductor microcavity. Detailed discussion of the noise source term will be given in Section III.

\section{SimUlation Results}

\section{A. Slab Optical Waveguides Containing Resonant Gain Nonlinearities}

We have performed a number of validation studies in order to establish the ability of our 1-D semiclassical model to correctly recover the nonlinear gain dynamics of a homogeneously broadened two-level saturable gain medium. Initially, we have compared our model with the density matrix theoretical results for the gain saturation in a two-level atomic medium [26]. The test structure used for these validation studies was a $15 \mu \mathrm{m}$ long, active GaAs slab with refractive index $n=3.59$. At the left boundary of the FDTD simulation space a continuous-wave sinusoidal source field given by (7), with the atomic transition frequency $\omega_{0}$ corresponding to the wavelength $\lambda=1.5 \mu \mathrm{m}$, is launched toward the slab. The population and polarization relaxation times of the damped resonant dipoles were set, respectively, to $T_{1}=100 \mathrm{ps}, T_{2}=50 \mathrm{fs}$, in accordance with the saturation condition at resonance, namely

$$
\frac{\wp^{2} E_{0}^{2} T_{1} T_{2}}{\hbar^{2}}>1
$$

where $\wp=1.0 \times 10^{-29} \mathrm{Cm}$ is the dipole coupling coefficient. The source field amplitude was varied from $E_{0}=5.0 \times$ $10^{6} \mathrm{Vm}^{-1}$ to $E_{0}=1.0 \times 10^{8} \mathrm{Vm}^{-1}$. The nonlinear gain dynamics and saturation are demonstrated in Fig. 1(a)-(d), where the electric field envelopes are plotted as a function of time with the initial electric field amplitude as a parameter. Initially, the increase in the excitation electric field amplitude inverts the two-level system and the population in the upper level provides gain which is visible from the initial region of amplification of the electric field envelope traces. The gain peaks and saturates afterwards in time due to the depletion of the population from the upper (excited) level of the two-level system. The higher the initial electric field amplitude is, the faster the nonlinear gain saturates with time and the sooner it reaches the stationary saturated value.

Furthermore, we have demonstrated quantitatively the agreement between the density matrix theory of saturation in a twolevel gain medium and the FDTD simulations. The simulation 


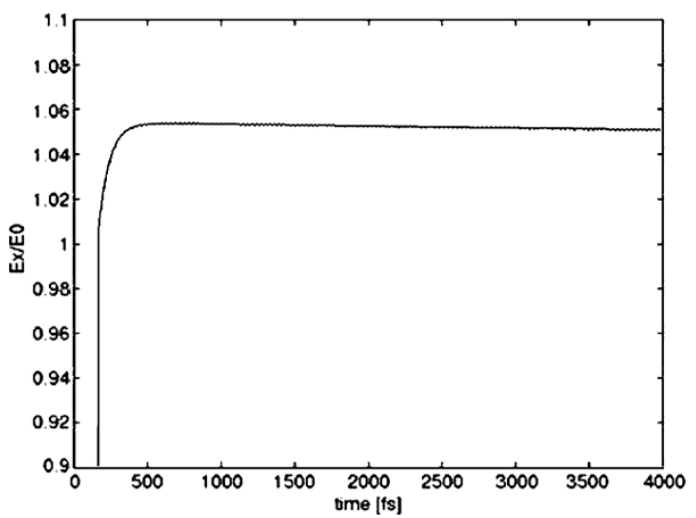

(a)

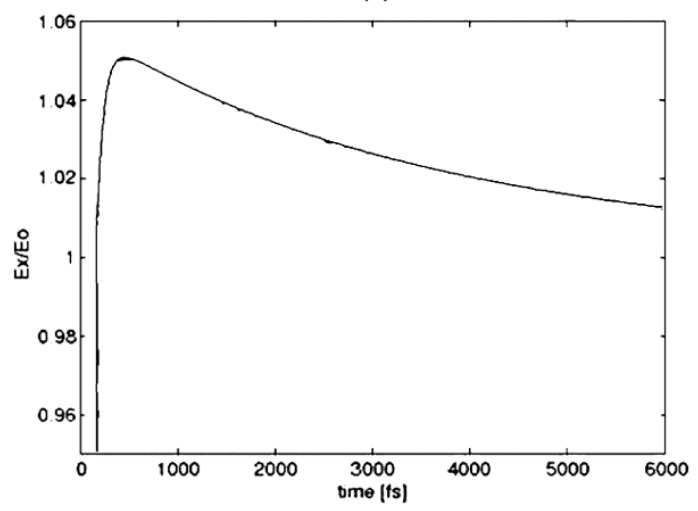

(b)

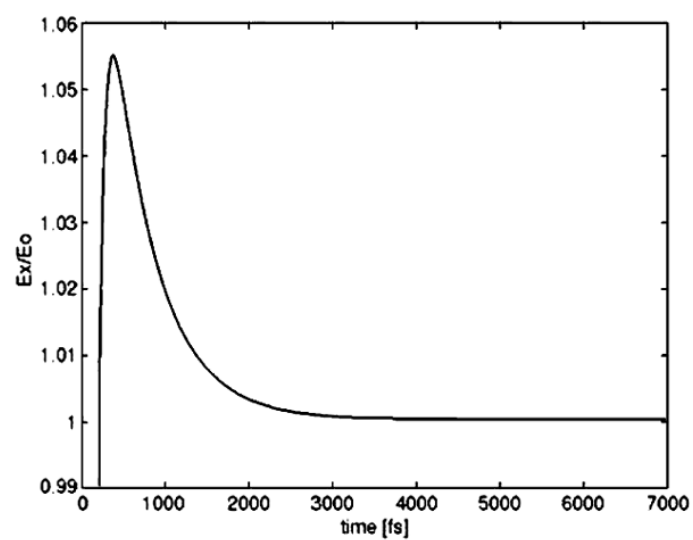

(c)

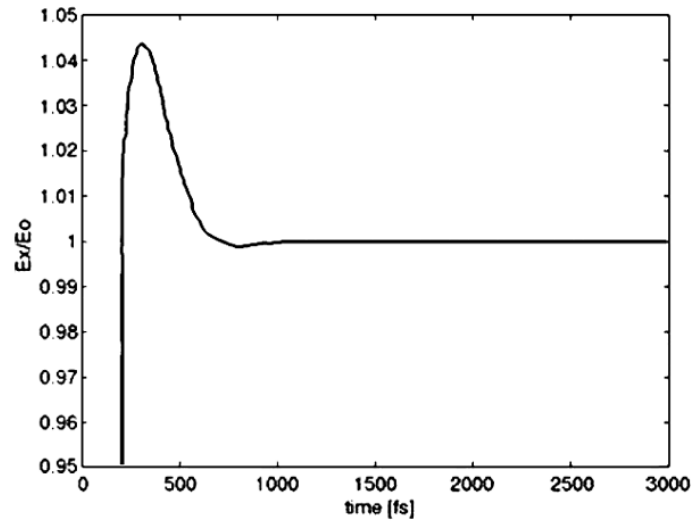

(d)

Fig. 1. Gain saturation dynamics of a homogeneously broadened two-level quantum system with increasing excitation field amplitude. The envelope of the fast-oscillating (with carrier frequency $\omega_{0}$ ) electric field is plotted. (a) $E_{0}=5.0 \times 10^{6} \mathrm{Vm}^{-1}$. (b) $E_{0}=2.0 \times 10^{7} \mathrm{Vm}-1$. (c) $E_{0}=5.0 \times 10^{7} \mathrm{Vm}^{-1}$. (d) $E_{0}=1.0 \times 10^{8} \mathrm{Vm}^{-1}$. The time trace of the electric field is sampled at the final cell of the simulation domain after the gain medium with $n=3.59$.

region for the latter is shown in Fig. 2(a); it was a gain medium slab with refractive index $n=1$ and length $L_{g}=9 \mu \mathrm{m}$, placed between two $3.0 \mu \mathrm{m}$ free-space regions $(n=1)$, thus giving a total FDTD simulation region length of $15 \mu \mathrm{m}$. The source field amplitude was set to $E_{0}=5.0 \times 10^{7} \mathrm{Vm}^{-1}$; and the relaxation times $T_{1}=10 \mathrm{ps}, T_{2}=10 \mathrm{fs}$ were chosen to satisfy the saturation condition at resonance (8). In Fig. 2(a), we show the spatial profile of the normalized electric field amplitude and the population inversion of the two-level system at the last $\left(48 \times 10^{6}\right)$ time step; and the relaxation of the population inversion toward the theoretical saturated value of 0.3 , as calculated from [26]

$$
\rho_{3 \mathrm{sat}}=\left(1+\left(\frac{E_{0}}{E_{s 0}}\right)^{2}\right)^{-1}, \quad E_{s 0}=\frac{\hbar}{\wp \sqrt{T_{1} T_{2}}}
$$

the electric field intensity saturation and the corresponding population inversion relaxation in the time domain are shown, respectively, in Fig.2(b) and (c). The unsaturated intensity gain coefficient can be calculated from the maximum intensity in Fig. 2(b), according to

$$
\gamma^{\text {unsat }}=\frac{\ln \left(\frac{I_{\max }}{I_{0}}\right)}{2 L_{g}}
$$

giving a value of $\gamma^{\text {unsat }}=2232.32 \mathrm{~m}^{-1}$. On the other hand, the saturation intensity $I=I_{\text {sat }}$ is the value of the signal intensity passing through the laser medium that will saturate the gain coefficient down to half of its small-signal (unsaturated) value [27]. It follows from the expression (10) for $\gamma^{\text {unsat }}$ as

$$
\gamma_{\mathrm{int}}^{\mathrm{sat}}=\frac{\gamma^{\mathrm{unsat}}}{1+\frac{I}{I_{\mathrm{sat}}}}
$$

Therefore, the normalized saturated field intensity is given by

$$
\frac{I_{\text {sat }}}{I_{0}}=\exp \left(\frac{\gamma^{\text {unsat }}}{2} L_{g}\right)
$$

which gives a value of $I_{\text {sat }} / I_{0} \sim 1.01$. This value is in very good agreement with the value inferred from Fig. 2(b). From (9), using the relaxation times chosen for this simulation, we can easily obtain the saturated value of the population inversion, namely $\rho_{3 \text { sat }} \approx 0.3$, which is the value that the population inversion converges to in Fig. 2(c).

These validation studies confirm the ability of our model to accurately recover the gain saturation dynamics of a homogeneously broadened two-level system.

\section{B. Langevin Formulation and FDTD Implementation}

Exploiting the quantum-classical correspondence in the presence of quantum noise, we have employed the Langevin formalism to consider the two-level atoms as having a few degrees of freedom in the heat bath of the radiation field which has an infinite number of degrees of freedom. The action of the many 

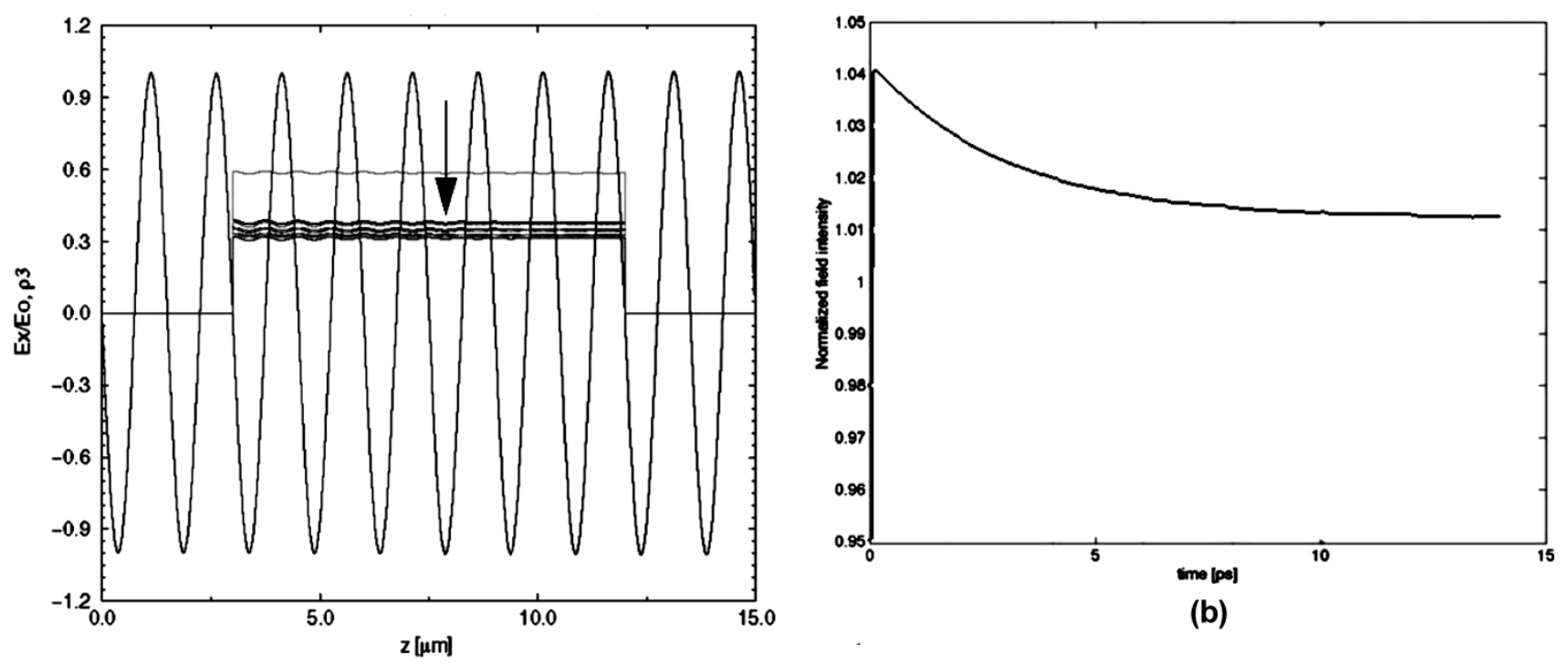

(b)

(a)

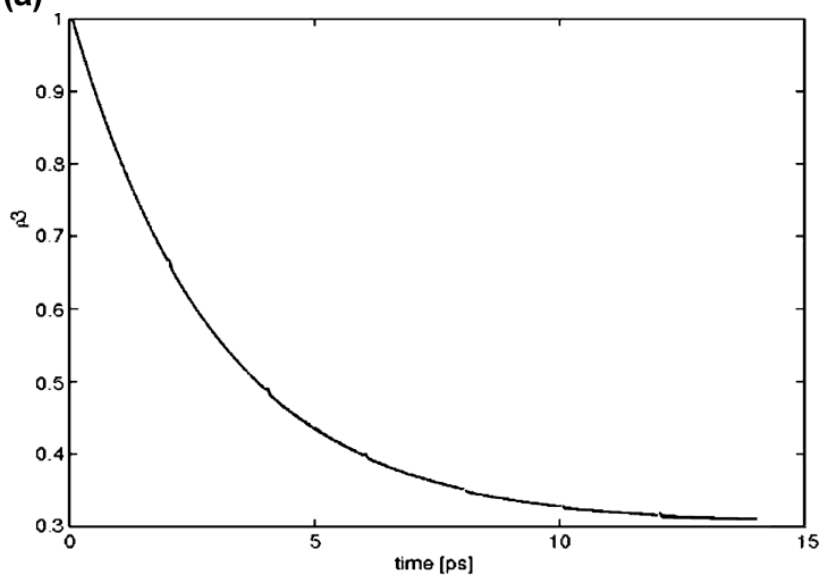

(c)

Fig. 2. (a) Spatial distribution of the sinusoidal electric field computed at the $48 \times 10^{6}$ time step and the population inversion profile at several subsequent time steps are shown. The FDTD results converge to the theoretically computed value of 0.3 with an increasing number of the simulation time steps (downwards pointing arrow). The initial population profile $\rho_{30}=+1$ was assumed in the gain medium slab; $n=1$ throughout the whole simulation domain. (b) Plot of the E-field envelope as a function of time. FDTD simulation of the electric field intensity saturation in time shows relaxation toward the theoretically predicted saturation value. (c) Population inversion of the two-level system in time shows relaxation toward the theoretically predicted saturation value.

variables of the "bath" on the system is to modify the deterministic equations of motion of the system by the inclusion of apparently random terms [21]. We have included the spontaneous emission in the semiclassical Maxwell-Bloch equations as a random electric field fluctuation term. This random term is added at each time step to the source field within the laser cavity (see, e.g., [24]). In particular, (2) was modified by adding a random $E$-field fluctuation $\delta E_{x}$ to the electric field at each time step, namely

$$
\frac{\partial\left(E_{x}(z, t)+\delta E_{x}(z, t)\right)}{\partial t}=-\frac{1}{\varepsilon} \frac{\partial H_{y}}{\partial z}-\frac{N_{a \wp}}{\varepsilon T_{2}} \rho_{1}+\frac{N_{a \wp} \omega_{0}}{\varepsilon} \rho_{2}
$$

A pseudorandom number generator (Box-Müller) method for generating random numbers with a normal (Gaussian) distribution from a uniform distribution over the interval $(0,1)[28]$ was used to implement a white Gaussian random noise term with a variance $\xi_{E}=\sigma^{2}=1.0 \times 10^{-3} \mathrm{~V}^{2} \mathrm{~m}^{-2}$ (standard deviation $\sigma=3.16 \times 10^{-2} \mathrm{Vm}^{-1}$ ). At each time step $j$, a random
$E$-field fluctuation was added to the currently computed $E$-field, according to

$$
E_{x}(z)_{j}=E_{x}(z)_{j}+\sqrt{-2 \xi_{E} \ln (a)} \cos (2 \pi b)
$$

where $a$ and $b$ are random numbers uniformly distributed in ( 0 , 1) interval. We note that white Gaussian noise has the following statistical properties:

$$
\begin{aligned}
\left\langle\delta E_{x}(z, t)\right\rangle & =0 \\
\left\langle\delta E_{x}(z, t) \delta E_{x}\left(z, t^{\prime}\right)\right\rangle & =\left\langle F_{E}^{*}(t) F_{E}^{*}\left(t^{\prime}\right)\right\rangle \\
& =\tilde{\xi}_{E} \delta\left(t-t^{\prime}\right)=\tilde{\xi}_{E} R_{s p} \delta\left(\frac{t-t^{\prime}}{\Delta t}\right) \\
& =\xi_{E} \delta\left(\frac{t-t^{\prime}}{\Delta t}\right)
\end{aligned}
$$

where the notation $\langle\ldots\rangle$ is used to represent the statistical ensemble averaging. We have denoted $\delta E_{x}(z, t)$ by $F_{E}^{*}(t)$; it has the dimension $\mathrm{Vm}^{-1}$. Also, in the second equality of (16), we have introduced the spontaneous emission rate $R_{s p}$ by rendering 
the $\delta$-function dimensionless, i.e., by normalizing it with respect to the time step $\Delta t=1 / R_{s p}$ which required using the well known distribution property $\delta(a x)=\delta(x) /|a|$. In the last equality of (16), we have introduced the variance of the $E$-field fluctuation $\xi_{E}$ in $\left[\mathrm{V}^{2} \mathrm{~m}^{-2}\right]$. The definition of the two-time correlation function of the random $E$-field fluctuation, (16), reflects the fact that the statistical fluctuations at time $t$ are uncorrelated with those at time $t^{\prime}$. Since the noise sources had essentially a Gaussian character, the first and second moments, given respectively by (15) and (16), completely determine the statistics of these sources.

By introducing dimensionless variables $\tilde{E}_{x}, \tilde{F}_{E}^{*}, s$, and $q$ according to the definitions

$$
\begin{aligned}
E_{x}(t) & =\frac{N_{a} \wp}{\varepsilon} \tilde{E}_{x}(s) \\
F_{E}^{*}(t) & =\frac{N_{a} \wp}{\varepsilon} \tilde{F}_{E}^{*}(s) \\
s & =\frac{t}{T_{2}} \\
q & =\frac{z}{c T_{2}}
\end{aligned}
$$

where $s$ and $q$ are dimensionless time and spatial coordinates, respectively, and $c=1 / \sqrt{\varepsilon \mu}$ is the speed of light in the medium, we can rewrite (13) as

$$
\frac{\partial \tilde{E}_{x}(s)}{\partial s}+\frac{\partial \tilde{F}_{E}^{*}(s)}{\partial s}=-\frac{\partial \tilde{H}_{y}}{\partial q}-\rho_{1}+\omega_{0} T_{2} \rho_{2}
$$

We can further introduce a new variable $\tilde{F}_{E}(t)$ having the dimension $s^{-1}$, according to

$$
\tilde{F}_{E}(t)=\frac{F_{E}^{*}(s)}{T_{2}}=\frac{\varepsilon}{N_{a} \wp T_{2}} F_{E}^{*}(t) .
$$

This new variable can be identified as the random noise term $F_{E}(t)$ in the stochastic rate equation [23] for which the two-time correlation function is given by

$$
\left\langle F_{E}(t) F_{E}\left(t^{\prime}\right)\right\rangle=R_{s p} \delta\left(t-t^{\prime}\right) .
$$

Substituting $\tilde{F}_{E}(t)$ from (22) into the equation and normalizing the $\delta$-function, one obtains the expression

$$
\left\langle F_{E}^{*}(t) F_{E}^{*}\left(t^{\prime}\right)\right\rangle=\frac{R_{s p}^{2} N_{a}^{2} \wp^{2} T_{2}^{2}}{\varepsilon^{2}} \delta\left(\frac{t-t^{\prime}}{\Delta t}\right) .
$$

By comparing this relation with (16), one can deduce

$$
\xi_{E}=\frac{R_{s p}^{2} N_{a}^{2} \wp^{2} T_{2}^{2}}{\varepsilon^{2}} .
$$

For the realistic set of parameters: the resonant dipole density $N_{a}=1 \times 10^{18} \mathrm{~m}^{-3}$, the dipole coupling coefficient $\wp=$ $4.8 \times 10^{-28} \mathrm{Cm}$ which has been calculated taking the exciton Bohr radius of GaAs as a separation between the charges in the dipole, and the dephasing time $T_{2}=70 \mathrm{fs}$, and for the variance $\xi_{E}=0.001 \mathrm{~V}^{2} \mathrm{~m}^{-2}$, we calculate the value $R_{s p}=$ $2.84026 \times 10^{10} \mathrm{~s}^{-1}$ for the spontaneous emission rate. This value is in reasonable agreement with the typical values quoted

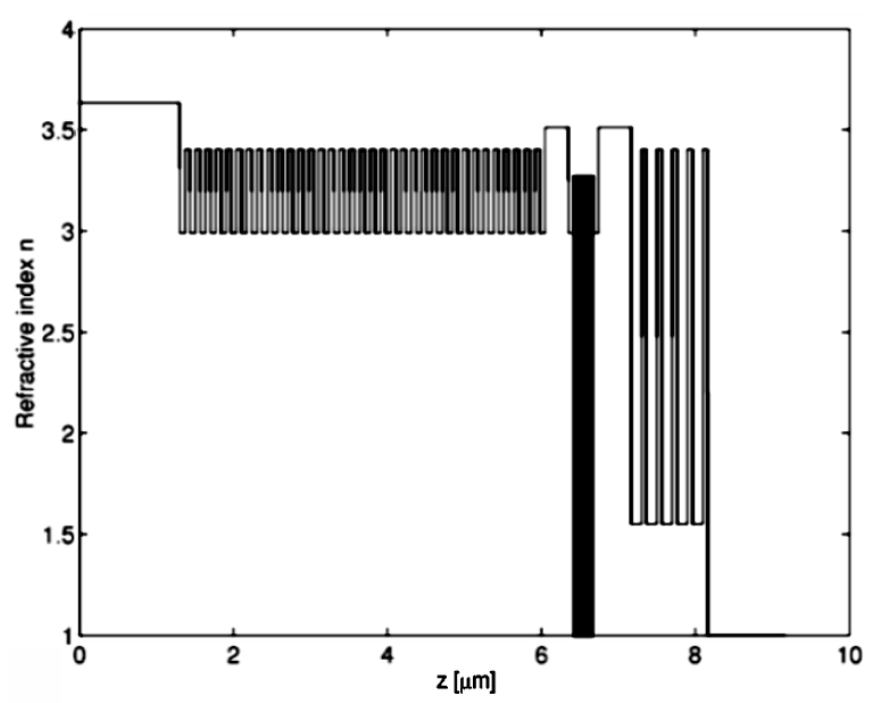

(a)

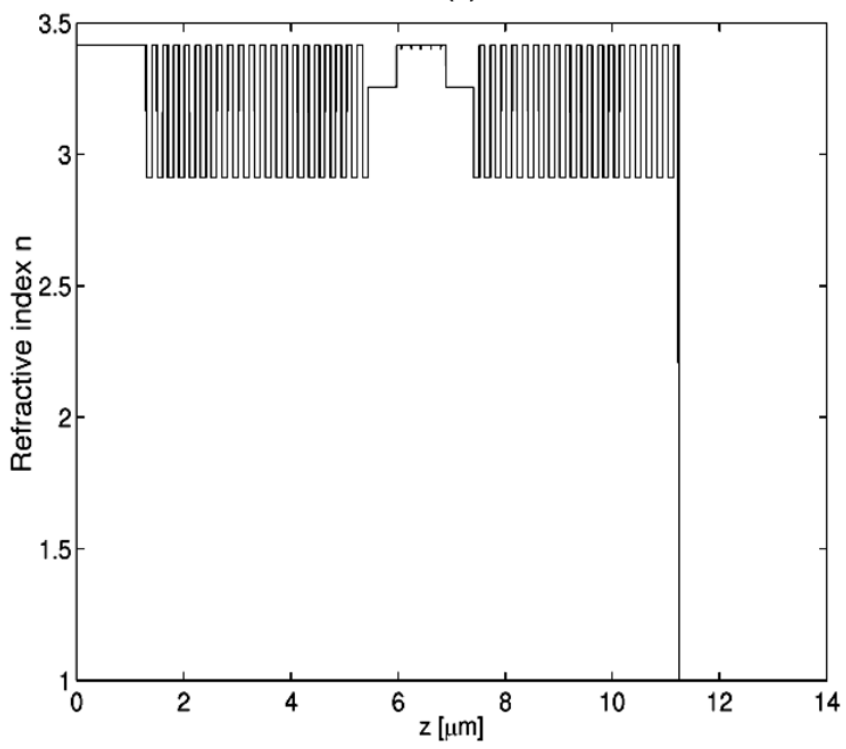

(b)

Fig. 3. Refractive index profile along the semiconductor microcavity structure. (a) DBRs: bottom-35.5 pairs $\mathrm{AlAs} / \mathrm{Al}_{0.3} \mathrm{Ga}_{0.7} \mathrm{As}$, top-five pairs $\mathrm{AlO} / \mathrm{Al}_{0.3} \mathrm{Ga}_{0.7} \mathrm{As}$. The Fabry-Pérot laser cavity is filled entirely with the $\mathrm{Al}_{0.5} \mathrm{Ga}_{0.5}$ As gain medium (the shaded area) characterized by $\lambda=0.85 \mu \mathrm{m}$, $n=3.2736$. (b) DBRs: bottom-20.5 pairs AlAs/GaAs; top-19 pairs AlAs/GaAs; $5-\lambda$ cavity with $6 \mathrm{Ga}_{0.63} \mathrm{In}_{0.37} \mathrm{AsN}_{0.012}$ quantum wells filled with the gain medium: $\lambda=1.29 \mu \mathrm{m}, n=3.4$.

in the literature (see, e.g., [1], [29]). Moreover, considering the advances in the semiconductor laser design to reduce noise, this value can be assumed as realistic.

\section{FDTD Simulations of the Quantum Noise in Active Semiconductor Microcavities}

The nonlinear gain medium was embedded in several realistic semiconductor microcavity geometries, two of which were designed at $\lambda=850 \mathrm{~nm}$ and $\lambda=1.29 \mu \mathrm{m}$ and are shown, respectively, in Fig. 3(a) and (b). Initially, we performed simulations on the structure shown in Fig. 3(a), whose cavity was entirely filled with a gain (initially inverted) medium. We applied solely random white Gaussian noise as the source term at each point within the cavity according to (14). The electric 


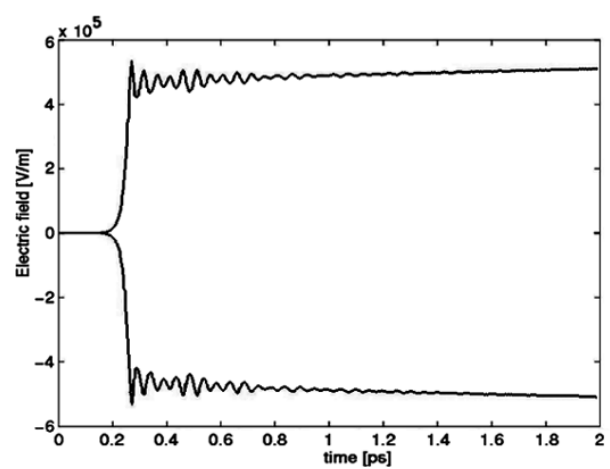

(a)

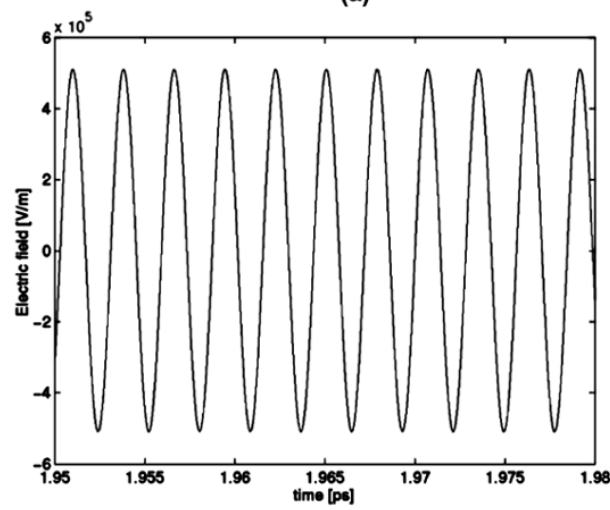

(b)

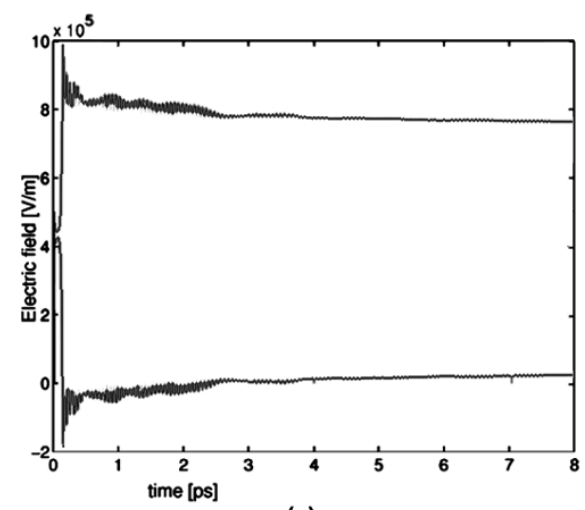

(c)

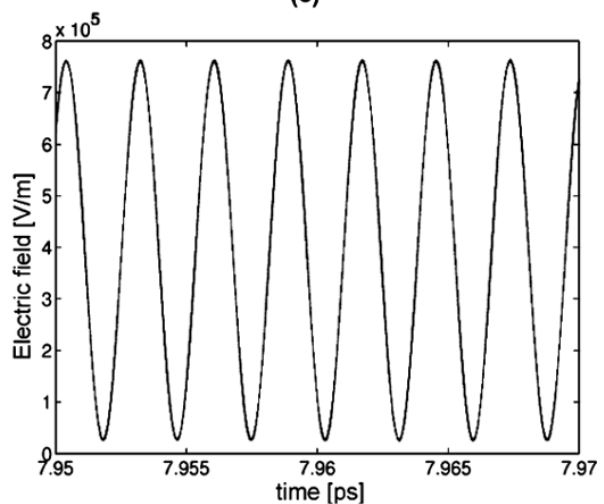

(d)

Fig. 4. FDTD computed time evolution of the electric field envelope at the output facet of the semiconductor microcavity shows the noise-induced build up of coherent oscillations (lasing). (a) $T_{1}=10 \mathrm{ps}, T_{2}=70 \mathrm{fs}$; (c) $T_{1}=1 \mathrm{~ns}, T_{2}=10 \mathrm{ps;} \wp=4.8 \times 10^{-28} \mathrm{Cm}$, with the resonant dipole density $N_{a}=1.0 \times$ $10^{24} \mathrm{~m}^{-3}$ being kept constant. Expanded time scale of the fast-oscillating (with carrier frequency $\omega_{0}$ ) part of the $E$-field time evolution in the steady-state regions of (a) and (c) that exhibit, respectively, single-mode oscillation at a frequency corresponding to the wavelength (b) $\lambda \approx 844.216 \mathrm{~nm}$, and (d) $\lambda \approx 849.999 \mathrm{~nm}$.

field evolution in time at the output facet of the laser was calculated. The parameter values used in this simulation were: $\wp=$ $4.8 \times 10^{-28} \mathrm{Cm}, n=3.2736, N_{a}=1 \times 10^{24} \mathrm{~m}^{-3}, T_{1}=10 \mathrm{ps}$, and $T_{2}=70 \mathrm{fs}$ with the initial field amplitude $E_{0}=700 \mathrm{Vm}^{-1}$. The FDTD simulations demonstrated that the multiple reflections from the DBR mirrors through the gain medium of the random $E$-field generated inside the resonant cavity are sufficient to build up the complete $E$-field from that noise field. This field growth, in turn, gives rise to coherent self-sustained oscillations once the threshold value is reached that start to radiate from the output facet after some initial build up time, as shown in Fig. 4(a). This FDTD predicted behavior is a demonstration of lasing when spontaneous emission is the only source of radiation (no external pumping mechanism is present). However, coherent oscillations build up because the stimulated emission starts to overtake the spontaneous emission, the latter being dynamically enhanced by the Fabry-Pérot microresonator and the multiple Bragg reflections from the mirror stacks. A similar situation is achieved physically in ultralow-threshold lasers [30]. However, a numerical treatment of those cases are beyond the scope of the present work.

For the values chosen, the saturation expression in (8) gives a value of 1.59. Therefore, the saturation condition was fulfilled in these simulations. However, because fulfillment of the inequality was weak, the resulting gain saturation was expected to occur only after very long simulation times. This behavior was confirmed by very long FDTD simulations that were run to 12 ps in time.
In Fig. 4(b), the final region of the trace in Fig. 4(a) is plotted on an expanded time scale. This result shows a single-mode sinusoidal oscillation at the wavelength $\lambda=844.216 \mathrm{~nm}$. Note that the final steady-state region was not yet reached. Nonetheless, the wavelength of the coherent oscillations thus obtained was in very good agreement with the designed wavelength. By appropriate choice of the relaxation times and the initial field amplitude, namely $T_{1}=1 \mathrm{~ns}, T_{2}=10 \mathrm{ps}, E_{0}=2.8 \times$ $10^{5} \mathrm{Vm}^{-1}$, and keeping the rest of the medium parameters the same as in the previous simulation, we achieved a much stronger inequality: $\Omega_{R}^{2} T_{1} T_{2} \sim 16241 \gg 1$, where $\Omega_{R}=\wp E_{0} / \hbar$ is the Rabi oscillation frequency. The results from this simulation are given in Fig. 4(c) and clearly show that the steady-state gain saturation was reached after 8 ps. From the sinusoidal steady-state region of Fig. 4(c), which is depicted in Fig. 4(d) on an expanded time scale, the lasing frequency or equivalently the lasing wavelength can be calculated. We obtained $\lambda=849.999 \mathrm{~nm}$. From Figs. 4(a) and (c) a sinusoidal perturbation of the field envelope is clearly discerned. We shall show further that these fluctuations from the steady state exhibit damped relaxation oscillations, performed on a very fast time scale. These fast relaxation oscillations are due to an intrinsic resonance in the nonlinear laser system, namely from the interplay between the resonance of the two-level quantum system and the Rabi flopping of the population and the cavity resonance. These rapidly-varying relaxation oscillations are a feature which is not predicted by the usual rotating wave and slowly varying envelope approximations. We should note that the present approach provides a fem- 

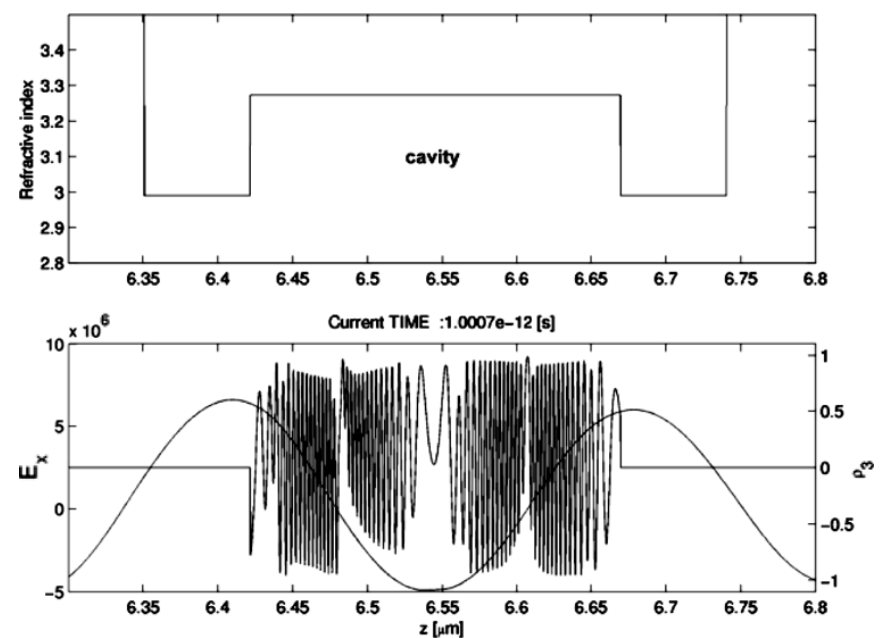

Fig. 5. Refractive index profile in the vicinity of the cavity shows the boundaries of the cavity (top). The spatial distribution of the intracavity standing wave $E$-field (left axis) and the corresponding population inversion density (right axis) are given (bottom) at $1 \mathrm{ps}$ after launching the initial random field within the cavity for the case shown in Fig. 4(c), (d). The population inversion exhibits the fast Rabi oscillations resulting in the observed relaxation oscillations in the electric field envelope in Fig. 4(a), (c) (bottom).

tosecond resolution of the subthreshold laser dynamics. It gives also an estimate of the lasing threshold and the time associated with the build up the coherent oscillations.

In Fig. 5, the spatial distribution of the intracavity electric field and the corresponding population inversion relaxation oscillations are shown for the same structure and parameters as in Fig. 4(c) and (d). The population inversion performed multiple Rabi-flops between the upper (excited) and lower (ground) state. The population oscillates symmetrically about a saturated value close to zero; and, consequently, the net gain and absorption in the system remain very close to each other, thereby leading to the gain saturation in time.

The structure at $\lambda=1.29 \mu \mathrm{m}$ was designed with six quantum wells within the cavity, the quantum wells being modeled by two-level systems whose population was initially inverted into the upper state. The system, therefore, represented a nonlinear gain medium. The initial population profile outside the quantum wells was assumed to be in the ground state, i.e., $\rho_{30}=-1$. Similar to the previous simulations, random noise was generated within the cavity according to (14). The values of the medium parameters were set equal to the values of the first simulation, namely: $\wp=4.8 \times 10^{-28} \mathrm{Cm}, N_{a}=1 \times 10^{24} \mathrm{~m}^{-3}, T_{1}=$ $10 \mathrm{ps}$, and $T_{2}=70 \mathrm{fs}$, while the refractive index $n=3.4$ was incorporated for the GaInNAs medium. The FDTD predicted build up of the coherent oscillations at the output VCSEL facet of this structure are shown Fig. 6(a). They eventually reached the steady-state gain saturation value at simulation times $\sim 16$ ps. The damped relaxation oscillations are also clearly visible. From the electric field values in the steady-state region, which are expanded in Fig. 6(b), the lasing wavelength was found to be $\lambda=1.289 \mu \mathrm{m}$, which is in very good agreement with the expected (designed) lasing wavelength. The relaxation-oscillation decay rate was also obtained from Fig. 6(a), giving a value of $\Gamma_{R} \sim 1.5 \times 10^{11} \mathrm{~s}^{-1}$.

In order to understand what the temporal behavior at the output facet should be, it is also important to know the spatial

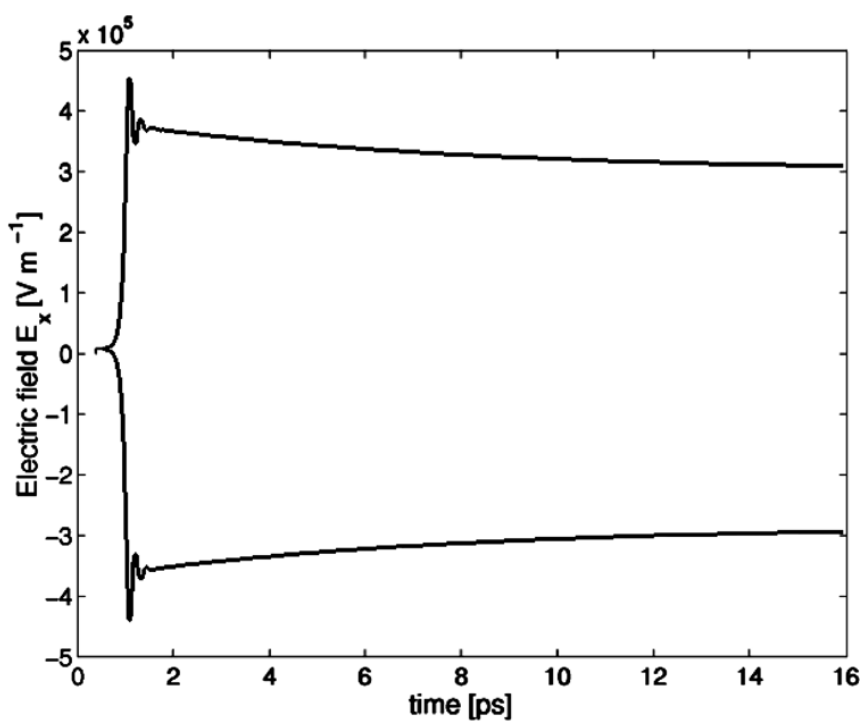

(a)

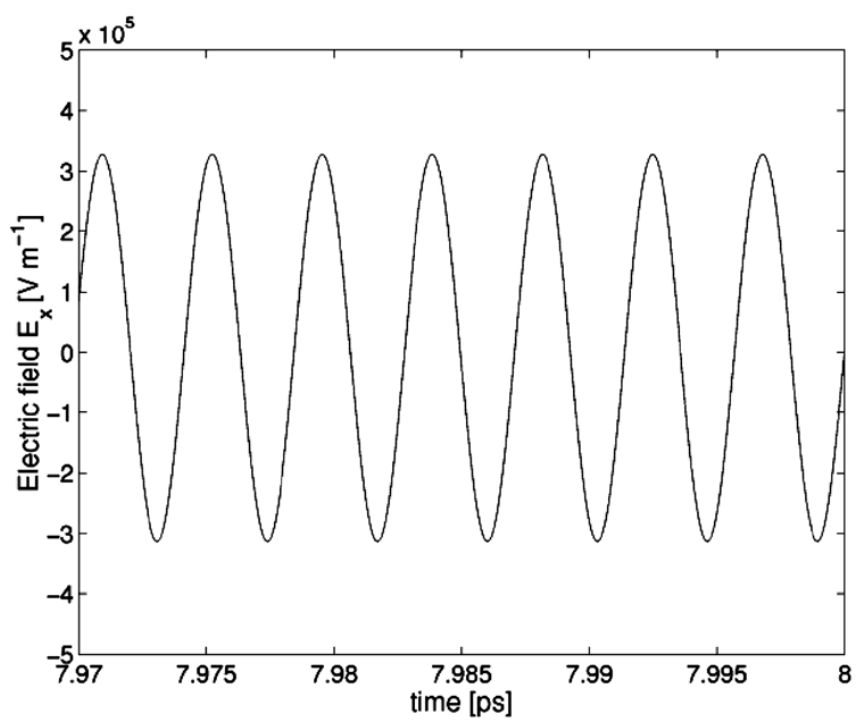

(b)

Fig. 6. Build up of coherent self-sustained oscillations at the output facet and the subsequent gain saturation are shown by plotting the FDTD predicted electric field envelope versus time. (a) Entire simulation time history. (b) Expanded view of the fast carrier frequency oscillations in the steady-state region of (a).

behavior. In Fig. 7, the spatial electric field and population inversion distributions at the simulation time $t=7.97 \mathrm{ps}$ are shown. The length of the simulation in time is long enough to ensure that the steady-state saturated value of the population inversion was reached, namely $\rho_{\text {sat }}=0.0025$. The fractional population difference of the two-level system relaxes to this value and oscillates very closely about it in time and space.

Having obtained the time trace of the electric field in Fig. 6(a), we performed a FFT on it to obtain the laser linewidth. The results for the so-obtained laser linewidth and the frequency spectrum are shown in expanded views in Figs. 8(a) and (b), respectively. Thesefiguresillustrate the presence of a superfineline structure. The main mode was centered at the angular frequency $\omega_{0}$ of the single mode oscillations (corresponding to $\lambda=1.289 \mu \mathrm{m}$ ). The wings of the main mode exhibit much weaker equally spaced 

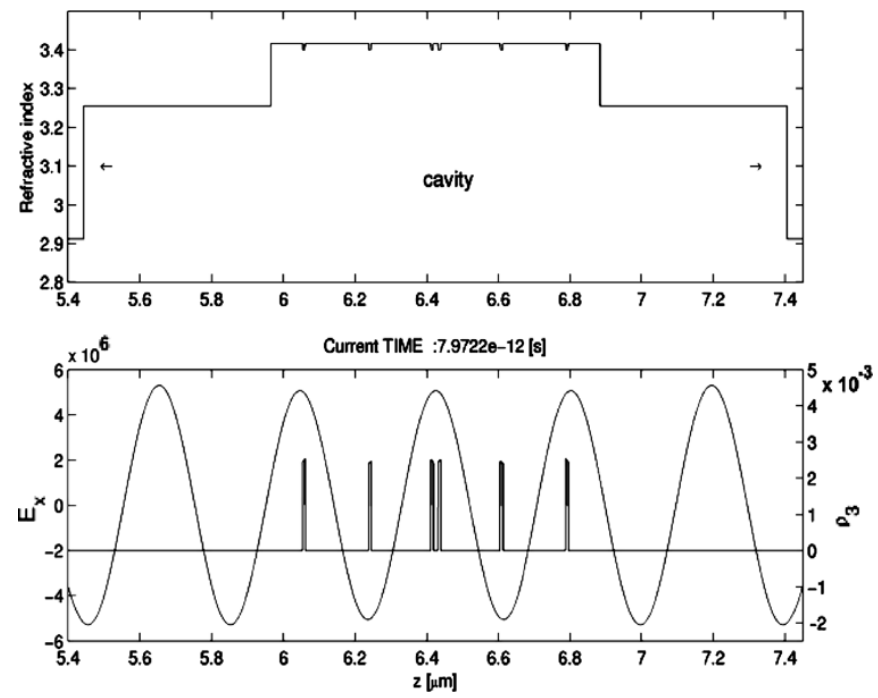

Fig. 7. Refractive index profile in the vicinity of the cavity shows the presence of six GaInNAs quantum wells (top). The spatial distribution of the E-field standing wave (left axis) and the corresponding saturated population inversion density in the cavity (right axis) are also given (bottom).

satellite peaks at $\omega_{0}+n \Omega_{\text {rel }}$. The side peaks arise from relaxation oscillations and form sidebands. The frequency of the relaxation oscillations can be determined from these figures, giving a value of $\Omega_{\mathrm{rel}} / 2 \pi \sim 2.54 \mathrm{THz}$. The expanded view in Fig. 8(b) shows the asymmetry in the amplitude of the peaks from both sides of the main peak. This asymmetry has been experimentally observed and interpreted in terms of the correlations between the amplitude and phase fluctuations [31].

\section{CONCLUSION}

We introduced a dynamical model for the nonlinear gain saturation and coherent response of active optical slab waveguides and semiconductor microcavities based on the numerical FDTD solution of the coupled Maxwell-Bloch equations for a twolevel quantum system. The model was successfully validated against the theoretical predictions of the density matrix method for the nonlinear gain saturation in a homogeneously broadened two-level system. Furthermore, the model equations were extended to include a Langevin model of quantum noise. The resulting numerical simulator was thus extended to account for the presence of spontaneous emission. We then numerically demonstrated the build up of coherent self-sustained oscillations solely from the noise background. We demonstrated the fast relaxation behavior of the electric field envelope which is not present in the usual rotating wave and slowly varying envelope approximations. The simulations provide an estimate for the coherence time of the laser emission. We showed that several important parameters can be extracted from the simulations and that they can be optimized by using numerical experiments on a variety of design geometries.

The introduced method represents a nonperturbative treatment of the nonlinearities in resonantly absorbing/amplifying media and thus does not require any of the standard, additional assumptions of small perturbations. Due to the generality of the full-vectorial coherent Maxwell-Bloch equations, the range of validity of the model extends to ultrashort optical pulses, with

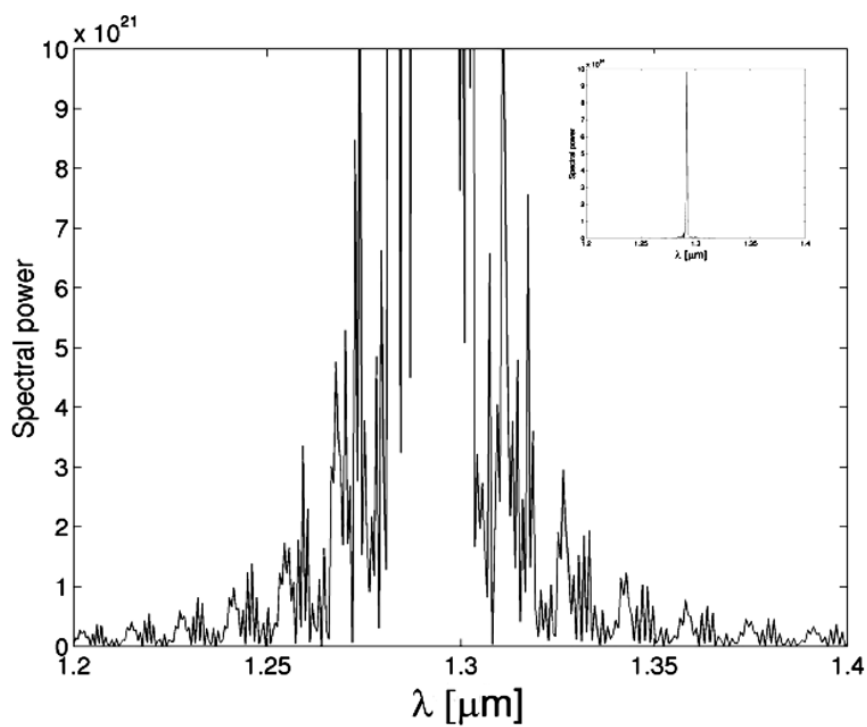

(a)

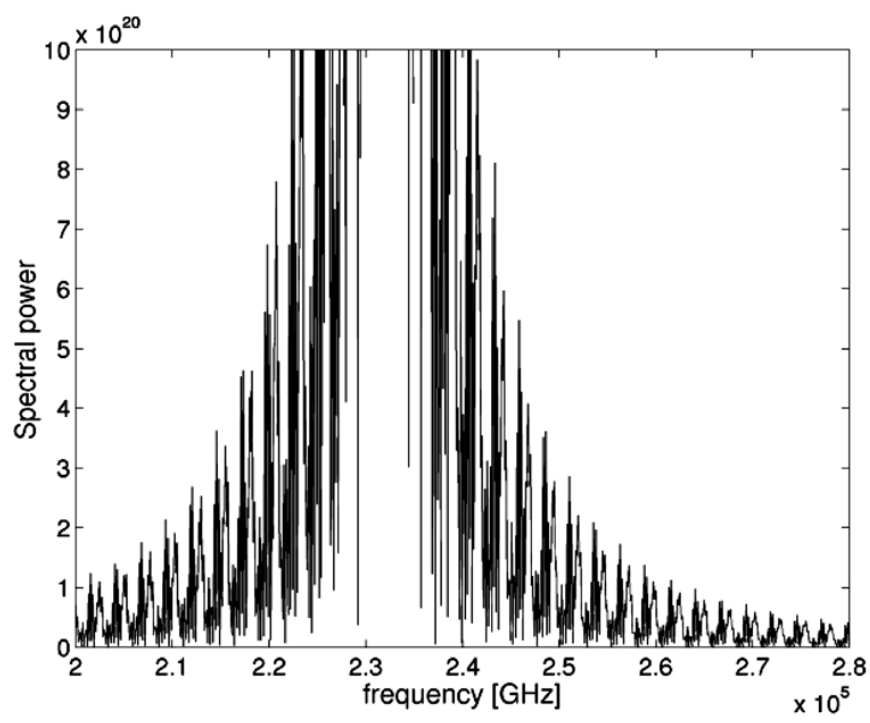

(b)

Fig. 8. Laser output spectrum was obtained as the FFT of the FDTD predicted E-field values at the output facet. (a) The values in the enhanced linewidth region (the inset represents the full scaleline shape). (b) Fequency spectrum of the relaxation oscillation sidebands.

pulse durations much less than the characteristic lifetimes in matter. Therefore, the limits of its applicability are beyond those of the widely used rate equation approach. This, in turn, provides the basis for a comprehensive analysis of the ultrafast dynamic response of semiconductor microcavities, and the basis for modeling fast saturable absorbers incorporated in integrated optoelectronic devices.

A significant advantage of this nonperturbative numerical approach is that it allows for a generalization to multidimensional and multilevel quantum system problems of the propagation and coherent interaction of optical pulses. This ability has been demonstrated by the authors in [32], [33] for two-dimensional geometries and a degenerate three-level quantum system. Further steps in the development of the model are currently under investigation. We are considering the inclusion of both spontaneous emission and the pumping dynamics in active optical 
slab waveguides and semiconductor microcavities in order to achieve a more realistic picture of the complex ultrafast nonlinear dynamics in these devices.

\section{ACKNOWLEDGMENT}

The authors gratefully acknowledge the CPU time on the 30-processor parallel Sun E6500 Enterprise server available under SHEFC grant VIDEOS at the Department of Electronics and Electrical Engineering. Significant speed-up has been achieved by parallelizing the FDTD-code, making the computations feasible within reasonable times.

\section{REFERENCES}

[1] G. P. Agrawal and N. K. Dutta, Semiconductor Lasers, 2nd ed. New York: Van Nostrand, 1993.

[2] J. Yao, G. P. Agrawal, P. Gallion, and C. Bowden, "Semiconductor laser dynamics beyond the rate-equation approximation," Opt. Commun., vol. 119, pp. 246-255, 1995.

[3] O. Hess and T. Kuhn, "Spatio-temporal dynamics of semiconductor lasers: Theory, modeling and analysis," Prog. Quantum Electron., vol. 20, pp. 85-178, 1996.

[4] C. Z. Ning, R. A. Indik, and J. V. Moloney, "Effective Bloch equations for semiconductor lasers and amplifiers," IEEE J. Quantum Electron., vol. 33, pp. 1543-1550, Sept. 1997.

[5] L. Allen and J. H. Eberly, Optical Resonance and Two-Level Atoms. New York: Dover, 1987, pp. 41-46.

[6] P. W. Milonni and J. H. Eberly, Lasers. New York: Wiley, 1988, pp. 250-252.

[7] P. N. Butcher and D. Cotter, The Elements of Nonlinear Optics. Cambridge, U.K.: Cambridge Univ. Press, 1991, pp. 216-217.

[8] F. Romstad, P. Borri, W. Langbein, J. Mørk, and J. M. Hvam, "Measurement of pulse amplitude and phase distortion in a semiconductor optical amplifier: from pulse compression to breakup," IEEE Photon. Technol. Lett., vol. 12, pp. 1674-1676, Dec. 2000.

[9] P. Michler, A. Lohner, and W. W. Rüle, "Transient pulse response of In ${ }_{0.2} G a_{0.8}$ As/GaAs microcavity lasers," Appl. Phys. Lett., vol. 66, pp. $1599-1601,1995$

[10] F. Jahnke, H. C. Schneider, and S. W. Koch, "Combined influence of design and carrier scattering on the ultrafast emission dynamics of quantum well microcavity lasers," Appl. Phys. Lett., vol. 69, pp. 1185-1187, 1996.

[11] S. Hughes, "Breakdown of the area theorem: carrier-wave Rabi flopping of femtosecond optical pulses," Phys. Rev. Lett., vol. 81, pp. 3363-3366, 1998

[12] F. Jahnke and S. W. Koch, "Many-body theory for semiconductor lasers," Phys. Rev. A, Gen. Phys., vol. 52, pp. 1712-1727, 1995.

[13] K. Henneberger and S. W. Koch, "Quantum kinetics of semiconductor light emission and lasing," Phys. Rev. Lett., vol. 76, pp. 1820-1823, 1996.

[14] H. F. Hofmann and O. Hess, "Quantum Maxwell-Bloch equations for spatially inhomogeneous semiconductor lasers," Phys. Rev. A, Gen. Phys., vol. 59, pp. 2342-2358, 1999.

[15] M. Kira, F. Jahnke, S. W. Koch, J. D. Berger, D. V. Wick, T. R. Nelson Jr., G. Khitrova, and H. M. Gibbs, "Quantum theory of nonlinear semiconductor microcavity luminescence explaining "Boser" experiments," Phys. Rev. Lett., vol. 79, pp. 5170-5173, 1997.

[16] M. Kira, F. Jahnke, and S. W. Koch, "Microscopic theory of excitonic signatures in semiconductor photoluminescence," Phys. Rev. Lett., vol. 81, pp. 3263-3266, 1998.

[17] _ "Quantum theory of secondary emission in optically excited semiconductor quantum wells," Phys. Rev. Lett., vol. 82, pp. 3263-3266, 1999

[18] R. W. Ziolkowski, J. M. Arnold, and D. M. Gogny, "Ultrafast pulse interactions with two-level atoms," Phys. Rev. A, Gen. Phys., vol. 52, pp. 3082-3094, 1995.

[19] G. Slavcheva, J. M. Arnold, and I. Wallace, "Dynamics of the coherent interaction of electromagnetic pulses in a two-level medium: FDTD study," in Tech. Dig. 15th Quantum Electronics and Photonics Conf. (IOP), Glasgow, U.K., Sept. 3-6, 2001, p. 66.
[20] M. Lax and W. H. Louisell, "Quantum noise. XII. Density-operator treatment of field and population fluctuations," Phys. Rev., vol. 185, pp. 568-591, 1969.

[21] C. W. Gardiner, Quantum Noise. Berlin, Germany: Springer-Verlag, 1991 , p. 42.

[22] D. Marcuse, "Computer simulation of laser photon fluctuations: Theory of single-cavity laser," IEEE J. Quantum Electron., vol. QE-20, pp. 1139-1148, Oct. 1984.

[23] G. Gray and R. Roy, "Noise in nearly-single-mode semiconductor lasers," Phys. Rev. A, Gen. Phys., vol. 40, pp. 2452-2462, 1989.

[24] A. Taflove, Computational Electrodynamics: The Finite-Difference Time-Domain Method, 2nd ed, A. Taflove and S. C. Hagness, Eds. Norwood, MA: Artech House, 2000, p. 779.

[25] A. C. Newell and J. V. Moloney, Nonlinear Optics. Reading, MA: Addison-Wesley, 1992, p. 233.

[26] A. Yariv, Quantum Electronics. New York: Wiley, 1989, p. 161.

[27] A. E. Siegman, Lasers. Mill Valley, CA: Univ. Sci. Books, 1986, p. 292.

[28] W. H. Press, B. P. Flannery, S. A. Teukolski, and W. T. Vetterling, Numerical Recipes in C: The Art of Scientific Computing. Cambridge, U.K.: Cambridge Univ. Press, 1992, p. 289.

[29] A. Hohl, H. J. C. van der Linden, and R. Roy, "Determinism and stochasticity of power-dropout events in semiconductor lasers with optical feedback," Opt. Lett., vol. 20, pp. 2396-2398, 1995.

[30] G. Björk, A. Karlsson, and Y. Yamamoto, "Definition of a laser threshold," Phys. Rev. A, Gen. Phys., vol. 50, pp. 1675-1680, 1994.

[31] K. Vahala, C. Harder, and A. Yariv, "Observation of relaxation resonance effects in the field spectrum of semiconductor lasers," Appl. Phys. Lett., vol. 42, pp. 211-213, 1983.

[32] G. Slavcheva, J. M. Arnold, I. Wallace, and R. W. Ziolkowski, "Coupled Maxwell-pseudospin equations for investigation of self-induced transparency effects in a degenerate three-level quantum system in two dimensions: Finite-difference time-domain study," Phys. Rev. A, Gen Phys., vol. 66, p. 063418, 2002.

[33] G. Slavcheva, J. M. Arnold, and R. W. Ziolkowski, "Ultrashort pulse lossless propagation through a degenerate three-level medium in nonlinear optical waveguides and semiconductor microcavities," IEEE $J$. Select. Topics Qunatum Electron., vol. 9, pp. 929-938, May/June 2003.

[34] S. Hughes, P. Borri, A. Knorr, F. Romstad, and J. M. Hvam, "Ultrashort pulse-propagation effects in a semiconductor optical amplifier: Microscopic theory and experiment," IEEE J. Select. Topics Quantum Electron., vol. 7, pp. 694-702, July/Aug. 2001.

[35] A. Knorr and S. Hughes, "Microscopic theory of ultrashort pulse compression and break-up in a semiconductor optical amplifier," IEEE Photon. Technol. Lett., vol. 8, pp. 782-784, Aug. 2001.

[36] G. Pompe, T. Rappen, and M. Wegner, "Transient response of an optically pumped short-cavity semiconductor laser," Phys. Rev. B, Condens. Matter, vol. 51, pp. 7005-7009, 1995.

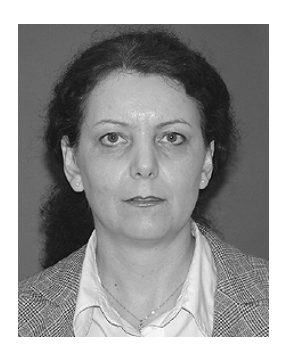

Gabriela M. Slavcheva received the M.Sc. degree in semiconductor physics from Sofia University, Sofia, Bulgaria, in 1983 and the Ph.D. degree in theoretical condensed matter physics from the Bulgarian Academy of Sciences, Sofia, Bulgaria, in 1997.

She has been active in the theoretical semiconductor physics, biophysics, quantum electronics, and nonlinear optics, including numerical modeling and simulation and high-performance computing since 1985. She spent a three and a half years leave in the Institute of Acoustics (C.N.R.), Rome, Italy and, subsequently, a six-month leave in the Physics Department, Chulalongkorn University, Bangkok, Thailand, as a Visiting Professor. Since 1999, she has been with the University of Glasgow, Glasgow, U.K., initially working on a NASA project in the Device Modeling Group, and thereafter joining the Optoelectronics Research Group where she carried out theoretical and computational studies of advanced optoelectronic devices (active optical waveguides and VCSELs). In 2004, she joined the Computational Quantum Electronics Group, University of Surrey, Surrey, U.K. 


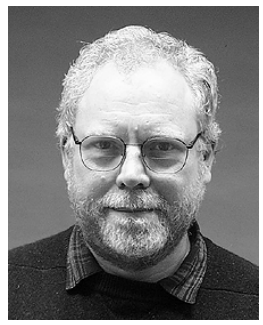

John M. Arnold received the B.Eng. degree in electronic engineering and the Ph.D. degree from the University of Sheffield, U.K., in 1968 and 1974, respectively.

He was a Postdoctoral Research Assistant at the Department of Electronic and Electrical Engineering, Queen Mary College, University of London, London, U.K., from 1974 to 1978. In 1978, he was appointed Lecturer at the Department of Electronic and Electrical Engineering, University of Nottingham, U.K. In 1985, he was appointed Lecturer in the Department of Electronics and Electrical Engineering, University of Glasgow, U.K., where has been Professor of Applied Electromagnetics since 1994. He was appointed Head of Department of Electronics and Electrical Engineering in April 2003. His research interests include mathematical methods in applications to optics and electromagnetic wave propagation, particularly in nonlinear guided-wave optics and semiconductor lasers.

Dr. Arnold is a Fellow of the Institute of Physics. He is a Member of the URSI Commission B and has served as the U.K. National Representative for URSI Commission B from 1991 to 1996.

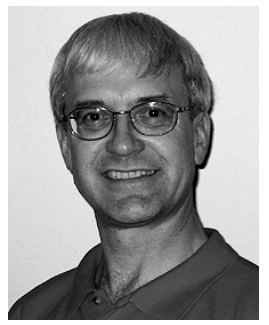

Richard W. Ziolkowski (M'97-SM'91-F'94) received the B.Sc. degree in physics (magna cum laude) with honors from Brown University, Providence, RI, in 1974 and the M.S. and Ph.D. degrees in physics from the University of Illinois at Urbana-Champaign, in 1975 and 1980, respectively.

He was a member of the Engineering Research Division, Lawrence Livermore National Laboratory, CA, from 1981 to 1990 , and served as the leader of the Computational Electronics and Electromagnetics Thrust Area for the Engineering Directorate, from 1984 to 1990. He joined the Department of Electrical and Computer Engineering, University of Arizona, Tuscon, as an Associate Professor in 1990, and was promoted to Full Professor in 1996. His research interests include the application of new mathematical and numerical methods to linear and nonlinear problems dealing with the interaction of acoustic and electromagnetic waves with realistic materials and structures.

Prof. Ziolkowski is a Member of Tau Beta Pi, Sigma Xi, Phi Kappa Phi, the American Physical Society, the Optical Society of America, the Acoustical Society of America, and Commissions B (Fields and Waves) and D (Electronics and Photonics) of URSI (International Union of Radio Science). He was an Associate Editor for the IEEE TRANSACTIONS ON ANTENNAS AND PROPAGATION from 1993 to 1998. He served as the Vice Chairman of the 1989 IEEE/AP-S and URSI Symposium in San Jose, and as the Technical Program Chairperson for the 1998 IEEE Conference on Electromagnetic Field Computation in Tucson. He served as a member of the IEEE AP-S Administrative Committee (ADCOM) from 2000 to 2002. He is currently serving as Co-Guest Associate Editor for an IEEE TRANSACTIONS ON ANTENNAS AND PROPAGATION Special Issue on Metamaterials. For the US URSI Society, he served as Secretary for Commission B (Fields and Waves) from 1993 to 1996 and as Chairperson of the Technical Activities Committee from 1997 to 1999, and as Secretary for Commission D (Electronics and Photonics) from 2001 to 2002. He is currently serving as a Member-at-Large of the U.S. National Committee (USNC) of URSI and as a member of the International Commission B Technical Activities Committee. He was a Co-Guest Editor of the 1998 Special Issue of the Journal of the Optical Society of America A featuring Mathematics and Modeling in Modern Optics. He was a Co-Organizer of the Photonics Nanostructures Special Symposia at the 1998, 1999, and 2000 OSA Integrated Photonics Research (IPR) Topical Meetings. He served as the Chair of the IPR subcommittee IV, Nanostructure Photonics, in 2001. He was awarded the Tau Beta Pi Professor of the Year Award in 1993 and the IEEE and Eta Kappa Nu Outstanding Teaching Award in 1993 and 1998. He also holds the title of Sensei with a Nidan rank in Matsunoryu Goshin Jujitsu and a Shodan rank in Kajukenbo. 